\title{
Important Aspects of Urine Sampling for Angiotensinogen Measurement: Time and Preservation Conditions in Healthy Individuals
}

\author{
Yoko Nishijima, ${ }^{1}$ Hiroyuki Kobori, ${ }^{2,3}$ Tadashi Sofue, ${ }^{1}$ Kumiko Kaifu, ${ }^{1}$ \\ Kumiko Moriwaki, ${ }^{1}$ Taiga Hara, ${ }^{1}$ Hirofumi Hitomi, ${ }^{2}$ Masakazu Kohno ${ }^{1}$ and \\ Akira Nishiyama ${ }^{2}$ \\ ${ }^{1}$ Department of Cardio Renal and Cerebro Vascular Medicine, Faculty of Medicine, Kagawa University, Kagawa, \\ Japan \\ ${ }^{2}$ Department of Pharmacology, Faculty of Medicine, Kagawa University, Kagawa, Japan \\ ${ }^{3}$ Departments of Medicine and of Physiology, and Hypertension and Renal Center of Excellence, Tulane \\ University Health Sciences Center, New Orleans, LA, USA
}

\begin{abstract}
Intrarenal renin-angiotensin system (RAS) plays an important role for the pathogenesis of renal injuries. Experimental studies have demonstrated that angiotensinogen levels in renal tissues reflect the activity of intrarenal RAS. However, dynamics of urinary angiotensinogen have not been investigated in detail. Therefore, we examined the preservation conditions of the measured values of urinary angiotensinogen concentrations and an ultradian rhythm of urinary angiotensinogen excretion in humans. Urine samples were collected from 24 healthy volunteers. The urinary concentrations of angiotensinogen were measured by using ELISA. Two different urine preservation conditions were examined. One cycle of freeze-and-thaw did not change the measured values of urinary angiotensinogen concentrations. Moreover, to keep urine samples at room temperature for 12 hours did not change the measured values of urinary angiotensinogen concentrations. Thus, preservation conditions do not change the measured values of urinary angiotensinogen concentrations. Regarding an ultradian rhythm, blood pressure and the urinary concentrations of angiotensinogen were measured at 09:00, 13:00, and 16:00. The averaged levels of blood pressure were similar over the time. The average of urinary angiotensinogen/creatinine $(\mathrm{Cr})$ ratios was $8.73 \pm 1.15 \mathrm{ng} / \mathrm{mg} \mathrm{Cr}$ at 09:00, $9.53 \pm 1.58 \mathrm{ng} / \mathrm{mg} \mathrm{Cr}$ at 13:00, and $8.58 \pm 1.26 \mathrm{ng} / \mathrm{mg} \mathrm{Cr}$ at 16:00. The urinary angiotensinogen excretion in healthy volunteers does not have an ultradian change during the daytime $(P=0.482)$. This may be another indication that the intrarenal RAS is independent of the systemic RAS. We have to pay attention to these findings in handling urine samples for measurements of angiotensinogen.
\end{abstract}

Keywords: angiotensinogen; clinical study; preservation condition; the ultradian rhythm; urine Tohoku J. Exp. Med., 2012 Dec, 228 (4), 333-339. 다이 2012 Tohoku University Medical Press

The renin-angiotensin system (RAS) is a hormone system that regulates blood pressure and fluid/electrolyte balance (Kobori et al. 2007). In recent years, there has been growing evidence that the local/tissue RAS in various tissues including the brain (Baltatu et al. 2000), the heart (Dell'Italia et al. 1997), vasculatures (Griendling et al. 1994; Danser et al. 1998) and kidneys (Navar et al. 2002) is independently regulated from the systemic RAS. Recent basic and clinical data demonstrated that the intrarenal RAS plays an important role in the development and the progression of hypertension and renal injury (Navar et al. 2011a). Elevated intrarenal angiotensin II (Ang II) levels were observed in renal functional damage and renal tissue injury.
Experimental studies demonstrated that the intrarenal Ang II levels were increased in several animal models of renal injury, including Dahl salt-sensitive rats on high salt diet (Kobori et al. 2003a; Kobori and Nishiyama 2004), spontaneously hypertensive rats (SHR) (Kobori et al. 2005) and diabetic nephropathy (Nagai et al. 2005).

Angiotensinogen (AGT) is the only known substrate for renin, which is the rate-limiting enzyme of the RAS. Because of the levels of AGT are close to the MichaelisMenten constant for renin, AGT levels can also control the RAS activity, and upregulation of AGT may lead to elevated angiotensin peptide levels (Gould and Green 1971; Brasier and Li 1996). Intrarenal AGT produced in proximal

Received June 14, 2012; accepted October 18, 2012. Published online November 7, 2012; doi: 10.1620/tjem.228.333.

Correspondence: Hiroyuki Kobori, M.D., Ph.D., Department of Pharmacology, Faculty of Medicine, Kagawa University, 1750-1

Ikenobe, Miki, Kita, Kagawa 761-0793, Japan.

e-mail: hkobori@kms.ac.jp 
tubular cells contributes to increased intrarenal Ang II in animal models (Kobori et al. 2001).

We have previously reported that urinary AGT excretion rate could be a novel biomarker for the activity of the RAS in kidney (Kobori et al. 2009, 2010). Novel sandwich ELISA for human angiotensinogen (Katsurada et al. 2007) made it possible for us to measure a large quantity of specimens over time.

It was reported that the systemic RAS had a circadian rhythm. For example, plasma renin activity (PRA), Ang II, and aldosterone rose in the early morning and decreased at night (Kala et al. 1973: Kawasaki et al. 1980). It was also reported that the cardiac RAS in the SHR rat had a circadian rhythm (Naito et al. 2002). Therefore, it is necessary to confirm that urinary AGT does not have an ultradian rhythm, because it is difficult to collect early morning urine samples or 24-hour urine samples in nonhospitalized patients. In addition, it is also important to examine whether preservation conditions may affect the measured values of urinary AGT. Therefore, this study was performed to demonstrate the following two points. 1) Preservation conditions would not change the measured values of urinary AGT concentrations. 2) Urinary AGT excretion in humans would not have an ultradian rhythm.

\section{Materials and Methods}

Subjects

The experimental protocol of this study was approved by the Institutional Review Board and by the Clinical and Translational Research Center at Tulane University and Kagawa University. Urine samples were collected from 24 healthy Japanese volunteers, recruited in Kagawa University, aged from 20 to 50 years old (Age: $32.4 \pm 5.8$ years old, Men/Women $=14 / 10$ ). Healthy volunteers having a history of diabetes mellitus, hypertension, urine disorder or metabolic syndrome were excluded. Those who were taking any medication or who had previously had any cardiovascular events were also excluded in this study.

Assays

Urine samples were centrifuged at 1,500 r.p.m. for $10 \mathrm{~min}$ at $4^{\circ} \mathrm{C}$. Urinary supernatants were pooled. Urinary AGT concentrations were measured by human AGT ELISA kits (IBL, Takasaki, Japan) as described previously (Katsurada et al. 2007). The coefficients of variation of the intra-assay and inter-assay of human AGT ELISA kits were 4.4 and $4.3 \%$, respectively. Urinary concentrations of creatinine (Cr) and albumin were measured by autoanalyzer (Hitachi, Hitachinaka, Japan). Urinary concentrations of AGT and albumin were normalized by urinary concentrations of $\mathrm{Cr}$.

\section{Blood pressure and other parameters}

Systemic blood pressure was measured three consecutive times after a rest for a minimum period of $15 \mathrm{~min}$. The averaged levels of blood pressure and urinary albumin/Cr ratio were summarized in Tables 1 and 2, respectively.

\section{Preservation conditions}

The urine samples were stored at $-80^{\circ} \mathrm{C}$ in previous studies
(Kobori et al. 2009, 2010). In this study, urine samples were stored at room temperature or deep freezer. Room temperature was $+20 \pm 3^{\circ} \mathrm{C}$. Deep freezer was $-80 \pm 3^{\circ} \mathrm{C}$. One group of samples (A) was stored for 1 week plus 36 hours. The other group of samples (B) was stored for 1 week and then defrosted and kept at room temperature for 12 hours. After 12 hours, it was frozen again at deep freezer for 24 hours. The samples of both groups were defrosted at the same time and the concentrations of AGT and Cr were measured.

One group of samples (C) was stored at deep freezer for 12 hours plus 1 week. The other group of samples (D) was kept at room temperature for 12 hours before freezing and then frozen at deep freezer for 1 week. The samples of both groups were defrosted at the same time and the concentrations of AGT and $\mathrm{Cr}$ were measured.

\section{Ultradian rhythm}

We examined an ultradian rhythm of urinary AGT excretion in healthy volunteers. Urine samples were collected at 09:00, 13:00, and 16:00, and all samples were stored at deep freezer. The concentrations of AGT, albumin, and $\mathrm{Cr}$ were measured. Blood pressure was also measured at 09:00, 13:00, and 16:00 as described previously (Kobori et al. 2009, 2010).

\section{Statistical analyses}

Comparisons of continuous variables between groups were performed using paired $t$-test or one-way repeated measures of analysis of variance and Dunnett's test. All data are presented as means \pm SEM. $P<0.05$ was considered significant. All computations, including data management and statistical analyses, were performed with the JMP software (SAS Institute).

\section{Results}

\section{Effects of preservation conditions on urinary $A G T$}

The average of measured values of urinary AGT concentrations (A) without thawing or (B) with thawing, was $13.14 \pm 5.06 \mathrm{ng} / \mathrm{ml}$ and $9.05 \pm 2.37 \mathrm{ng} / \mathrm{ml}$, respectively. The average of ratio (B/A) was $0.926 \pm 0.087$ (Fig. $1 \mathrm{~A}, P=$ $0.401,95 \%$ confidence interval: $0.747-1.105$ ). These data indicate that one cycle of freeze-and-thaw did not change the measured values of urinary AGT concentrations.

The average of measured values of urinary AGT concentrations stored (C) at deep freezer and kept (D) at room temperature before freezing, was $13.60 \pm 5.24 \mathrm{ng} / \mathrm{ml}$ and $11.79 \pm 3.51 \mathrm{ng} / \mathrm{ml}$, respectively. The average of ratio (D/ C) was $1.068 \pm 0.061$ (Fig. $1 \mathrm{~B}, P=0.280,95 \%$ confidence interval: 0.941-1.194). These data indicate that to keep urine samples at room temperature for 12 hours before freezing did not change the measured values of urinary AGT concentrations.

\section{Ultradian rhythm of urinary $A G T$}

The averaged levels of blood pressure were similar over the time $(122.8 \pm 2.6 / 74.1 \pm 1.4 \mathrm{mmHg}$ at $09: 00,116.1$ $\pm 2.6 / 71.1 \pm 1.6 \mathrm{mmHg}$ at $13: 00$, and $119.3 \pm 4.2 / 74.8 \pm 1.9$ $\mathrm{mmHg}$ at 16:00) (Table 1). The averaged levels of urinary albumin/Cr ratio were also similar over the time $(5.08 \pm$ $0.43 \mathrm{mg} / \mathrm{g} \mathrm{Cr}$ at 09:00, $5.26 \pm 0.36 \mathrm{mg} / \mathrm{g} \mathrm{Cr}$ at 13:00, and $4.89 \pm 0.35 \mathrm{mg} / \mathrm{g} \mathrm{Cr}$ at 16:00) (Table 2). Urinary AGT 
A

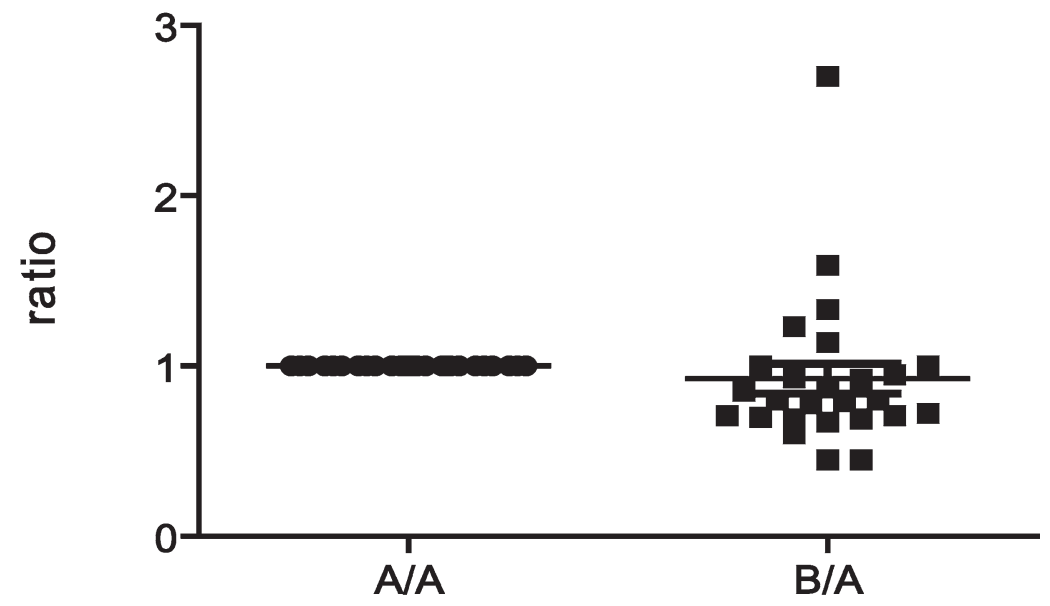

B

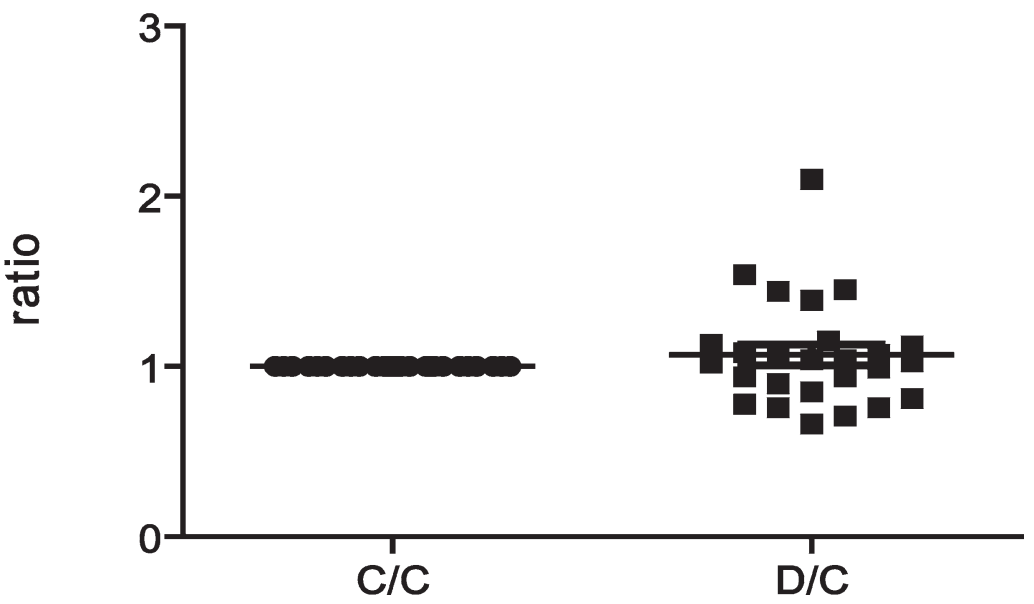

Fig. 1. Effects of Preservation Conditions on Urinary AGT in Healthy Volunteers.

A. The average of measured values of urinary AGT concentrations of without thawing (A) or with thawing (B) was $13.14 \pm 5.06 \mathrm{ng} / \mathrm{ml}$ and $9.05 \pm 2.37 \mathrm{ng} / \mathrm{ml}$, respectively $(n=24)$. The average of ratio $(\mathrm{B} / \mathrm{A})$ was $0.926 \pm 0.087(P=$ $0.401,95 \%$ confidence interval: $0.747-1.105)$. These data indicate that one cycle of freeze-and-thaw did not change the measured values of urinary AGT concentrations.

B. The average of measured values of urinary AGT concentrations stored at deep freezer $(C)$ and kept at room temperature before freezing (D) was $13.60 \pm 5.24 \mathrm{ng} / \mathrm{ml}$ and $11.79 \pm 3.51 \mathrm{ng} / \mathrm{ml}$, respectively $(n=24)$. The average of ratio $(\mathrm{D} / \mathrm{C})$ was $1.068 \pm 0.061(P=0.280,95 \%$ confidence interval: $0.941-1.194)$. These data indicate that to keep urine samples at room temperature for 12 hours before freezing did not change the measured values of urinary AGT concentrations.

Table 1. Averaged levels of blood pressure (mean \pm SEM)

\begin{tabular}{cccc}
\hline & $09: 00$ & $13: 00$ & $16: 00$ \\
\hline SBP $(\mathrm{mmHg})$ & $122.8 \pm 2.6$ & $116.1 \pm 2.6$ & $119.3 \pm 4.2$ \\
DBP $(\mathrm{mmHg})$ & $74.1 \pm 1.4$ & $71.1 \pm 1.6$ & $74.8 \pm 1.9$ \\
\hline
\end{tabular}

Table 2. Averaged levels of urinary albumin/Cr ratio (mean $\pm \mathrm{SEM})$.

\begin{tabular}{cccc}
\hline & $09: 00$ & $13: 00$ & $16: 00$ \\
\hline Urinary albumin $/ \mathrm{Cr}$ ratio $(\mathrm{mg} / \mathrm{g} \mathrm{Cr})$ & $5.08 \pm 0.43$ & $5.26 \pm 0.36$ & $4.89 \pm 0.35$ \\
\hline
\end{tabular}


excretion in healthy volunteers did not have an ultradian change during the daytime (Fig. 2A, $10.66 \pm 1.60 \mathrm{ng} / \mathrm{ml}$ at $09: 00,10.66 \pm 1.63 \mathrm{ng} / \mathrm{ml}$ at $13: 00$, and $8.68 \pm 1.35 \mathrm{ng} / \mathrm{ml}$ at $16: 00, P=0.078)$. Urinary $\mathrm{Cr}$ excretion in healthy volunteers did not have an ultradian change during the daytime (Fig. $2 \mathrm{~B}, 129.3 \pm 11.6 \mathrm{mg} / \mathrm{dl}$ at $09: 00,120.1 \pm 9.8 \mathrm{mg} / \mathrm{dl}$ at $13: 00$, and $114.5 \pm 11.2 \mathrm{mg} / \mathrm{dl}$ at $16: 00, P=0.498$ ). Urinary AGT/Cr ratio in healthy volunteers did not have an ultradian change during the daytime (Fig. $2 \mathrm{C}, 8.73 \pm 1.15 \mathrm{ng} / \mathrm{mg}$ at $09: 00,9.53 \pm 1.58 \mathrm{ng} / \mathrm{mg}$ at $13: 00$, and $8.58 \pm 1.26 \mathrm{ng} /$ $\mathrm{mg}$ at $16: 00, P=0.482$ ). Then, the relative ratio was calculated for each volunteer on the basis of measured values of urinary AGT at 09:00. Urinary AGT/Cr ratio (relative ratio) did not have an ultradian change during the daytime (Fig. 2D, $1.00 \pm 0.00$ at 09:00, $1.12 \pm 0.09$ at 13:00, and 1.02 \pm 0.11 at $16: 00, P=0.400)$.

\section{Discussion}

Others (Kim et al. 2011a, b; Lee et al. 2011; Mao et al. 2012) and we (Kobori et al. 2009, 2010) recently reported that urinary AGT excretion rate could be a novel biomarker for the activity of the RAS in kidney. However, dynamics of urinary AGT in humans have not been fully elucidated yet. Our data in this study clearly demonstrate that 1) preservation conditions would not change the measured values of urinary AGT concentrations and that 2) urinary AGT excretion in humans would not have an ultradian change during the daytime. We have to pay attention to these findings in handling urine samples for AGT measurements.

It is well known that renin is excreted into urine (Navar et al. 2011b) and that inactive renin is cryoactivated (Castrop et al. 2010). Therefore, the freeze-and-thaw cycles of urine samples may activate renin in urine samples and cleave intact AGT into angiotensin I and des-angiotensin I AGT. However, the antibodies used in the AGT ELISA in this study recognize both intact AGT and des-angiotensin I AGT (Katsurada et al. 2007). Therefore, even though the cryoactivated renin in urine samples fragmented intact AGT into angiotensin I and des-angiotensin I AGT, the measured values of urinary AGT concentrations would not change in this AGT ELISA. In order to address this possibility, a new ELISA for intact AGT is required for further analyses.

PRA and Ang II had a circadian rhythm (Kala et al. 1973; Kawasaki et al. 1980). For PRA and Ang II, the highest value was detected early in the morning and the lowest value was detected in the evening (Kala et al. 1973; Kawasaki et al. 1980). The value of PRA was also changed by the posture and elevated by standing (Kala et al. 1973; Kawasaki et al. 1980). The prorenin and angiotensin converting enzyme (ACE) had a circadian rhythm as well (Cugini and Lucia 2004). The circadian rhythm of mRNA expression of the RAS components (renin, AGT, ACE, Ang II type 1a receptor, and Ang II type 2 receptor) was investigated in hearts of SHR and the Wister-Kyoto (WKY) rats (Naito et al. 2002). The cardiac RAS expression in the SHR had a circadian rhythm and varied greatly compared with the WKY (Naito et al. 2002). The reason why systemic Ang II has a circadian rhythm was discussed (Naito et al. 2002). The average minimums in renal plasma flow and glomerular filtration rate occurred between 04:00 and 05:00, and urinary excretion of sodium was also at its minimum early in the morning (Wesson 1964). Epinephrine, cortisol, and plasma aldosterone also have a circadian rhythm similar to Ang II. These factors may account for the circadian rhythm of the systemic RAS. However, the circadian rhythm of kidney/urinary Ang II has not been reported yet. Thus, up to this point, we have come up with no good explanation for the reason why the urinary angiotensinogen has no ultradian rhythm even though the systemic Ang II has a circadian rhythm. This may be another indication that the intrarenal RAS is independent of the systemic RAS (Kobori et al. 2007).

Intrarenal Ang II is independently regulated from the systemic RAS and is not evaluated by PRA or plasma Ang II (Navar et al. 2011b). Intrarenal RAS activity is regulated by changes in local AGT levels and urinary excretion of AGT reflects the expression of intrarenal Ang II (Kobori et al. 2007). It is reported that urinary AGT levels is increased in hypertensive patients (Kobori et al. 2009, 2010) and in IgA nephropathy patients (Konishi et al. 2011). However, it is still unclear whether intrarenal RAS has an ultradian rhythm or not. From the results of this study, urinary AGT levels were unchanged at different time points. Therefore, urinary $\mathrm{AGT} / \mathrm{Cr}$ ratio in spot urine samples can be used to estimate the urinary AGT excretion over 24 hours. Urinary AGT concentrations in spot urine samples are dependent on hydration/dehydration status. Using AGT/Cr ratio, we are able to correct dilutions or concentrations by taking the urinary $\mathrm{Cr}$ concentrations into account. Our study showed that urinary AGT did not change by meals and the normal labor in healthy volunteers. Therefore, we can measure the urinary AGT/Cr ratio at 09:00, 13:00, and 16:00, which are usually during office hours at the hospital. It is still being discussed whether urinary albumin has a circadian rhythm, however, we recently reported that urinary albumin levels in spot morning urine samples are lower than those in the daytime (Kaifu et al. 2011). Albumin is filtered through the capillaries of the glomerulus. On the other hand, intrarenal AGT is not filtered through the capillaries of the glomerulus (Kobori et al. 2003b) and intrarenal AGT is produced in proximal tubular cells (Kobori et al. 2001). Thus, urinary AGT may be unaffected by renal plasma flow or glomerular filtration rate, which is different from the urinary albumin, and the intrarenal RAS activity could be similar over time in healthy volunteers.

One limitation of this study is that urine samples were could not be collected in the early morning or at night because the subjects were nonhospitalized healthy volunteers. Urinary AGT excretion may or may not change in the early morning or at night. In addition, we cannot rule out the possibility that the intrarenal RAS activity could have an ultradian rhythm in some pathophysiological con- 

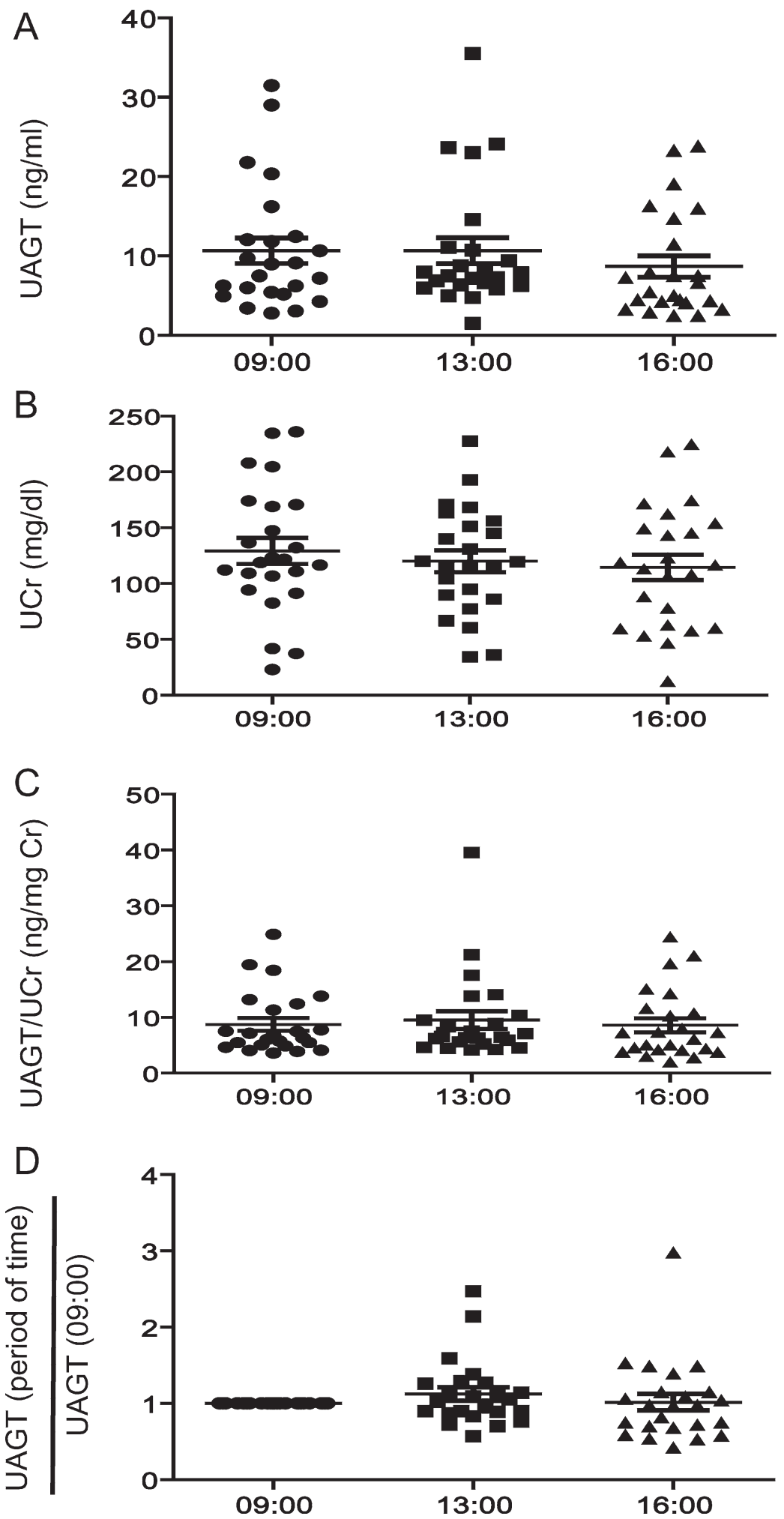

Fig. 2. Effects of Ultradian Rhythm of Urinary AGT in Healthy Volunteers.

A. Urinary AGT excretion in healthy volunteers did not have an ultradian rhythm $(10.66 \pm 1.60 \mathrm{ng} / \mathrm{ml}$ at 09:00, $10.66 \pm$ $1.63 \mathrm{ng} / \mathrm{ml}$ at 13:00, and $8.68 \pm 1.35 \mathrm{ng} / \mathrm{ml}$ at $16: 00, P=0.078, n=24)$. B. Urinary $\mathrm{Cr}$ excretion in healthy volunteers did not have an ultradian rhythm $(129.3 \pm 11.6 \mathrm{mg} / \mathrm{dl}$ at $09: 00,120.1 \pm 9.8 \mathrm{mg} / \mathrm{dl}$ at $13: 00$, and $114.5 \pm 11.2 \mathrm{mg} / \mathrm{dl}$ at $16: 00, P=0.498, n=24)$. C. Urinary AGT/Cr ratio in healthy volunteers did not have an ultradian rhythm $(8.73 \pm 1.15$ $\mathrm{ng} / \mathrm{mg}$ at $09: 00,9.53 \pm 1.58 \mathrm{ng} / \mathrm{mg}$ at $13: 00$, and $8.58 \pm 1.26 \mathrm{ng} / \mathrm{mg}$ at $16: 00, P=0.482, n=24)$. D. The relative ratio was calculated for each volunteer on the basis of measured values of urinary AGT at 09:00. Urinary AGT/Cr Ratio (Relative Ratio) did not have an ultradian rhythm $(1.00 \pm 0.00$ at 09:00, $1.12 \pm 0.09$ at 13:00, and 1.02 \pm 0.11 at 16:00, $P=0.400, n=24)$. 
ditions including hypertension, diabetic nephropathy, and chronic kidney diseases. Further studies are in progress to clarify these issues through evaluating the ultradian rhythm of urinary AGT in patients with continuous proteinuria or hematuria who will be hospitalized for a renal biopsy.

\section{Conclusion}

Our data in healthy volunteers clearly demonstrate that 1) preservation conditions would not change the measured values of urinary AGT concentrations and that 2) urinary AGT excretion in humans would not have an ultradian change during the daytime. We have to pay attention to these findings in handling urine samples for AGT measurements.

\section{Acknowledgments}

The authors acknowledge excellent technical assistance from Aya Masuda, BS, and Yoshiko Fujita, BS (Kagawa University). The authors also appreciate Ms. Natsuko Ishii (Kagawa University, Kagawa, Japan) for her help to prepare the manuscript.

\section{Grants}

National Institute of Diabetes and Digestive and Kidney Diseases (R01DK072408), National Center for Research Resources (P20RR017659), National Heart, Lung, and Blood Institute (R01HL026371), Louisiana Board of Regents (Research Commercialization and Educational Enhancement Program for Louisiana State University/Tulane University Health Sciences Centers' Clinical and Translational Research Education and Commercialization Program), Tulane University (Research Enhancement Fund Phase I and Phase II), Mitsui Life Social Welfare Foundation (Grant-in-Aid for Medical Research), and Kagawa University (Strategic Research Fund, Special Encouragement for Research, and Grant-in-Aid from Alumni Association)

\section{Conflict and Interest}

All authors have no conflict of interest in this study.

\section{References}

Baltatu, O., Silva, J.A. Jr., Ganten, D. \& Bader, M. (2000) The brain renin-angiotensin system modulates angiotensin II-induced hypertension and cardiac hypertrophy. Hypertension, 35, 409-412.

Brasier, A.R. \& Li, J. (1996) Mechanisms for inducible control of angiotensinogen gene transcription. Hypertension, 27, 465-475.

Castrop, H., Hocherl, K., Kurtz, A., Schweda, F., Todorov, V. \& Wagner, C. (2010) Physiology of kidney renin. Physiol. Rev., 90, 607-673.

Cugini, P. \& Lucia, P. (2004) Circadian rhythm of the renin-angiotensin-aldosterone system: a summary of our research studies. Clin. Ter., 155, 287-291.

Danser, A.H., Admiraal, P.J., Derkx, F.H. \& Schalekamp, M.A. (1998) Angiotensin I-to-II conversion in the human renal vascular bed. J. Hypertens., 16, 2051-2056.

Dell'Italia, L.J., Meng, Q.C., Balcells, E., Wei, C.C., Palmer, R., Hageman, G.R., Durand, J., Hankes, G.H. \& Oparil, S. (1997) Compartmentalization of angiotensin II generation in the dog heart. Evidence for independent mechanisms in intravascular and interstitial spaces. J. Clin. Invest., 100, 253-258.
Gould, A.B. \& Green, D. (1971) Kinetics of the human renin and human substrate reaction. Cardiovasc. Res., 5, 86-89.

Griendling, K.K., Minieri, C.A., Ollerenshaw, J.D. \& Alexander, R.W. (1994) Angiotensin II stimulates NADH and NADPH oxidase activity in cultured vascular smooth muscle cells. Circ. Res., 74, 1141-1148.

Kaifu, K., Nogami, H., Kawakami, K., Moritoki, M., Nishioka, S., Nishijima, Y., Sofue, T., Hara, T., Kobori, H., Nishiyama, A. \& Kohno, M. (2011) First-morning-void urine samples are recommended for the measurements of albuminuria in outpatients. Hypertens. Res., 34 (suppl), 155.

Kala, R., Fyhrquist, F. \& Eisalo, A. (1973) Diurnal variation of plasma angiotensin II in man. Scand. J. Clin. Lab. Invest., 31, 363-365.

Katsurada, A., Hagiwara, Y., Miyashita, K., Satou, R., Miyata, K., Ohashi, N., Navar, L.G. \& Kobori, H. (2007) Novel sandwich ELISA for human angiotensinogen. Am. J. Physiol. Renal Physiol., 293, F956-960.

Kawasaki, T., Ueno, M., Uezono, K., Matsuoka, M., Omae, T., Halberg, F., Wendt, H., Taggett-Anderson, M.A. \& Haus, E. (1980) Differences and similarities among circadian characteristics of plasma renin activity in healthy young women in Japan and the United States. Am. J. Med., 68, 91-96.

Kim, S.M., Jang, H.R., Lee, Y.J., Lee, J.E., Huh, W.S., Kim, D.J., Oh, H.Y. \& Kim, Y.G. (2011a) Urinary angiotensinogen levels reflect the severity of renal histopathology in patients with chronic kidney disease. Clin. Nephrol., 76, 117-123.

Kim, Y.G., Song, S.B., Lee, S.H., Moon, J.Y., Jeong, K.H., Lee, T.W. \& Ihm, C.G. (2011b) Urinary angiotensinogen as a predictive marker in patients with immunoglobulin A nephropathy. Clin. Exp. Nephrol., 15, 720-726.

Kobori, H., Alper, A.B. Jr., Shenava, R., Katsurada, A., Saito, T., Ohashi, N., Urushihara, M., Miyata, K., Satou, R., Hamm, L.L. \& Navar, L.G. (2009) Urinary angiotensinogen as a novel biomarker of the intrarenal renin-angiotensin system status in hypertensive patients. Hypertension, 53, 344-350.

Kobori, H., Harrison-Bernard, L.M. \& Navar, L.G. (2001) Expression of angiotensinogen mRNA and protein in angiotensin II-dependent hypertension. J. Am. Soc. Nephrol., 12, 431-439.

Kobori, H., Nangaku, M., Navar, L.G. \& Nishiyama, A. (2007) The intrarenal renin-angiotensin system: from physiology to the pathobiology of hypertension and kidney disease. Pharmacol. Rev., 59, 251-287.

Kobori, H. \& Nishiyama, A. (2004) Effects of tempol on renal angiotensinogen production in Dahl salt-sensitive rats. Biochem. Biophys. Res. Commun., 315, 746-750.

Kobori, H., Nishiyama, A., Abe, Y. \& Navar, L.G. (2003a) Enhancement of intrarenal angiotensinogen in Dahl salt-sensitive rats on high salt diet. Hypertension, 41, 592-597.

Kobori, H., Nishiyama, A., Harrison-Bernard, L.M. \& Navar, L.G. (2003b) Urinary angiotensinogen as an indicator of intrarenal Angiotensin status in hypertension. Hypertension, 41, 42-49.

Kobori, H., Ozawa, Y., Suzaki, Y. \& Nishiyama, A. (2005) Enhanced intrarenal angiotensinogen contributes to early renal injury in spontaneously hypertensive rats. J. Am. Soc. Nephrol., 16, 2073-2080.

Kobori, H., Urushihara, M., Xu, J.H., Berenson, G.S. \& Navar, L.G. (2010) Urinary angiotensinogen is correlated with blood pressure in men (Bogalusa Heart Study). J. Hypertens., 28, $1422-1428$.

Konishi, Y., Nishiyama, A., Morikawa, T., Kitabayashi, C., Shibata, M., Hamada, M., Kishida, M., Hitomi, H., Kiyomoto, H., Miyashita, T., Mori, N., Urushihara, M., Kobori, H. \& Imanishi, M. (2011) Relationship between urinary angiotensinogen and salt sensitivity of blood pressure in patients with IgA nephropathy. Hypertension, 58, 205-211.

Lee, Y.J., Cho, S., Kim, S.R., Jang, H.R., Lee, J.E., Huh, W., Kim, D.J., Oh, H.Y. \& Kim, Y.G. (2011) Effect of losartan on proteinuria and urinary angiotensinogen excretion in non- 
diabetic patients with chronic kidney disease. Postgrad. Med. $J ., 87,664-669$.

Mao, Y.N., Liu, W., Li, Y.G., Jia, G.C., Zhang, Z., Guan, Y.J., Zhou, X.F. \& Liu, Y.F. (2012) Urinary angiotensinogen levels in relation to renal involvement of Henoch-Schonlein purpura in children. Nephrology (Carlton), 17, 53-57.

Nagai, Y., Yao, L., Kobori, H., Miyata, K., Ozawa, Y., Miyatake, A., Yukimura, T., Shokoji, T., Kimura, S., Kiyomoto, H., Kohno, M., Abe, Y. \& Nishiyama, A. (2005) Temporary angiotensin II blockade at the prediabetic stage attenuates the development of renal injury in type 2 diabetic rats. J. Am. Soc. Nephrol., 16, 703-711.

Naito, Y., Tsujino, T., Fujioka, Y., Ohyanagi, M. \& Iwasaki, T. (2002) Augmented diurnal variations of the cardiac renin- angiotensin system in hypertensive rats. Hypertension, 40, 827-833.

Navar, L.G., Harrison-Bernard, L.M., Nishiyama, A. \& Kobori, H. (2002) Regulation of intrarenal angiotensin II in hypertension. Hypertension, 39, 316-322.

Navar, L.G., Kobori, H., Prieto, M.C. \& Gonzalez-Villalobos, R.A. (2011a) Intratubular renin-angiotensin system in hypertension. Hypertension, 57, 355-362.

Navar, L.G., Prieto, M.C., Satou, R. \& Kobori, H. (2011b) Intrarenal angiotensin II and its contribution to the genesis of chronic hypertension. Curr. Opin. Pharmacol., 11, 180-186.

Wesson, L.G. Jr. (1964) Electrolyte excretion in relation to diurnal cycles of renal function. Medicine (Baltimore), 43, 547-592. 